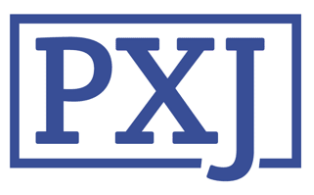

Patient Experience Journal

Volume 8 | Issue 1

Article 21

2021

\title{
Testing of Patients First in a real-world setting, as a patient experience accreditation tool for hospitals and clinics
}

\author{
Carlos Bezos \\ Instituto paraa la Experiencia del Paciente \\ Rosa M. Salazar \\ Hospital de Guadarrama \\ María Caballero \\ Hospital de Guadarrama
}

Follow this and additional works at: https://pxjournal.org/journal

Part of the Health and Medical Administration Commons, Health Policy Commons, Health Services Administration Commons, and the Health Services Research Commons

\section{Recommended Citation}

Bezos C, Salazar RM, Caballero M. Testing of Patients First in a real-world setting, as a patient experience accreditation tool for hospitals and clinics. Patient Experience Journal. 2021; 8(1):184-191. doi:

$10.35680 / 2372-0247.1432$.

This Case Study is brought to you for free and open access by Patient Experience Journal. It has been accepted for inclusion in Patient Experience Journal by an authorized editor of Patient Experience Journal. 


\section{Testing of Patients First in a real-world setting, as a patient experience accreditation tool for hospitals and clinics}

\section{Cover Page Footnote}

This article is associated with the Policy \& Measurement lens of The Beryl Institute Experience Framework (https://www.theberylinstitute.org/ExperienceFramework). You can access other resources related to this lens including additional PXJ articles here: http://bit.ly/PX_PolicyMeasure 


\title{
Testing of Patients First in a real-world setting, as a patient experience accreditation tool for hospitals and clinics \\ Carlos Bezos, Instituto paraa la Experiencia del Paciente, carlos.bezos@iexp.es \\ Rosa M. Salazar, Hospital de Guadarrama, rosam.salazan@salud.madrid.org \\ María Caballero Nahum, Hospital de Guadarrama, maria.caballero@salud.madrid.org
}

\begin{abstract}
Many healthcare providers are developing patient experience strategies and investing in this area. Yet, patients have no means to know if a hospital is following proper patient experience standards. For this reason, it is important to certify that hospitals and clinics follow own a patient experience policy and apply patient experience standards. This is the reason why the accreditation Patients First was developed. The goal of this study is to test the accreditations' feasibility in a real-life environment. The accreditation was tested at the Guadarrama Hospital, nearby Madrid in Spain. A mixed team between Guadarrama staff and the Institute for Patient Experience (IEXP) was set up in order to test each of the seven dimensions of the accreditation with a triple data gathering: documentation, participant observation, workshops with patients and also with staff. The certification has proven its utility to improve existing practices, as well as an instrument to set up new working lines in patient experience. The test also seems to prove that the certification's methodology is robust form a conceptual and operative point of view. Further research is needed to measure direct impact on patients.
\end{abstract}

\section{Keywords}

Patient experience, patient-centered care, quality of care, measurement, qualitative methods

\section{Introduction}

There is plenty of scientific evidence on the impact of efforts to improve patient experience on different levels, of healthcare: clinical outcomes, quality of life and cost reduction. ${ }^{1,2}$ The challenge is to find the means to ensure that hospitals that want to follow patient experience policies follow suitable standards that produce results and include the patients' perspective. Certainly, there are already instruments like the Hospital Consumer Assessment of Healthcare provider and Systems (HCAHPS) and other surveys like the Picker Institute questionnaires or the IEXPAC scale for the evaluation of the chronic disease patient experience. ${ }^{3}$ Scores and questionnaires gather the patient's voice, and this is useful for hospital management as long as patients have been included in their design and/or validation. Although strong evidence exists on the positive impact of scores and the correlation of scores and results, ${ }^{4}$ there has been criticism on scores and questionnaires mainly because social factors (literacy, ethnic background, etc.) and community factors seem to have a higher influence on how scores are answered than initially assumed., 5

Regarding healthcare accreditations, literature acknowledges their high impact. 7, 8, 9,10,11 Most of the studies describe in the literature have been carried out in the USA, but the mentioned impact can be observed also on an international level: A study in Hong Kong shows that an accreditation exercise may enhance patient experience. ${ }^{12}$ More interesting due to the broadness of the study is this comparison carried out in Germany, Switzerland, UK, the Netherlands, the USA and Australia, in which the authors concluded that "collecting patient experience data is of great importance for a comprehensive assessment of medical care quality. ${ }^{13}$ On the negative side, there are critical literature reviews that question the correlation between quality impact and clinical effectiveness. ${ }^{14}$

\section{The Patients First accreditation}

The Patients First accreditation is a tool to ensure that a given healthcare provider follows a systematic process to capture, transform and measure patient experience fulfilling adequate patient experience standards. The desired result is a process of continuous improvement of the patient experience. The idea for Patients First arose out of the experience of one of the authors of this article while in charge of the quality department at a fertility clinic. This author noted that the standard satisfaction questionnaires were focused on the information that the clinic wanted to know about its delivery (cleanliness, understanding of information, medical performance, friendliness of staff, etc.) rather than on the issues that 
were more important to the actual experience and needs of the patients of the clinic (which were usually more dominated by issues such as anxiety, stigma, pain, emotional relief, etc.). Literature widely supports partially this particular insight. ${ }^{15-20}$ It became apparent that a more comprehensive evaluation - one that gathers data on the aspects that the patients consider most relevant as well as basic data on the institution's performance - might contribute significantly to ensure that hospitals and clinics really fulfill patient experience standards in the most objective way possible. To this end, the Institute for Patient Experience (IEXP) developed 2016 a set of standards organised along a set of dimensions of patient experience that includes elements to address the missing aspect.

IEXP was founded 2016 with the aim of helping healthcare providers to understand and improve patient experience, as well as to support them in including patient insights in their strategies and processes. The accreditation Patients First was one of the first tools developed by IEXP in order to achieve these goals.

The goals of the certification for hospitals are to ensure that the healthcare provider has professional tools and methods to capture the voice and the experience of the patient in order to analyze and understand it. Further to guarantee that the healthcare provider uses this information to transform and improve patient experience. Later to include patients in the transformation of patient experience with validation, consultation and or co-creation tools. Another goal is to enable patient empowerment. Also to ensure a patient-centered culture on all the levels of the organization and along the continuum of care. And finally to transform the above listed points into an ongoing and replicable patient experience process with tools and protocols.

As a result of this approach, patients would benefit from this patient centered culture and an ongoing patient experience process in several ways. First, unmet needs would be identified in a systematic way. Second, patient's voice would be taken into account for the improvement of these unmet needs, as well as in the development of processes and protocols. Third, the patient experience process would open a space for specific solutions for important questions that normally do not get enough management attention, like emotional needs or patient empowerment.

Beyond these advantages for patients there would also be advantages for the healthcare provider, far beyond the positive effect that getting an accreditation might have on their public image. First, improved patient experience is likely to have an impact on clinical outcome, quality of life and also in cost reduction - as outlined above in the discussion of the literature. Second, an ongoing patient experience process supported by a patient centered culture provides the means for going beyond single actions and ensure these benefits for the hospital in a systematic and measurable way.

The present communication covers three main points. First, it describes the testing of the Patients First accreditation that specifically evaluates patient experience in a real hospital. The testing was undertaken) during 2017 in Guadarrama Hospital, a hospital of 125 beds for functional and cognitive rehabilitation near Madrid (Spain). Second, it discusses a significant amount of insights that result from a consistent process with regular patient experience capture and involvement. These insights can be used for more effective quality, innovation, clinical and even commercial strategies. Third, it argues on the base of this evidence that patient involvement and co-creation produce a far greater acceptance of protocols and services, as well as increased satisfaction. ${ }^{21-24}$

\section{Research goals}

In order to test the accreditation's feasibility, a pilot project was set up at the Guadarrama hospital with the following goals: The first goal was to test the certification structure by dimensions. As a second goal the study aimed to test the certification process triangulating information from documentation, participant observation/interviews, as well as patient and employee workshops. And finally, the third goal consisted in testing the kind of outcomes the accreditation process produces for patients and the organization

\section{Dimensions of the Patients First accreditation}

There are many approaches and definitions about what patient experience means. The Beryl Institute - a leading reference of Patient Experience worldwide - recommends that each healthcare organization should build and create its own definition of patient experience. ${ }^{25}$ The Beryl Institute itself defines patient experience as "The sum of all interactions, shaped by an organization's culture, that influence patient perceptions across the continuum of care." 26 A similar definition was created by the Cleveland Clinic, another world reference in patient experience: "Putting patients first requires more than world-class clinical care - it requires care that addresses every aspect of a patient's encounter with Cleveland Clinic, including the patient's physical comfort, as well as their educational, emotional, and spiritual needs." 27 In Cleveland's definition we can observe a precise approach with specific elements such as comfort, educational or emotional needs.

The American perspective that both The Beryl Institute and Cleveland Clinic are part of is very much conditioned by the nature of the US healthcare system as a private system were patients are viewed as consumers. Therefore, customer satisfaction is central, and this is why interactions (touch points) are the key elements valuated 
by the standard surveys, such as hospital consumer assessment of healthcare providers and systems (HCAHPS), which have an impact in hospital reimbursement.

European approaches are different and define patient experience by the dimensions this experience is composed of. For instance, for the British National Healthcare System (NHS), patient experience is made of 8 dimensions among which we can find respect for patients' values and preferences, co-ordination of care, emotional support or continuity. ${ }^{28}$ Another European reference institution, the Picker Institute, also organizes patient experience based on dimensions that are similar to those of the NHS, with some differences like the involvement of patients in decision making of the participation of family members and care givers. ${ }^{29}$

Despite the growing importance of patient experience as a tool for healthcare management and for patient wellbeing, patients have no means to find out if a healthcare provider is following a robust and founded patient experience strategy and process of the provider is just making isolated improvement actions or - worse - just marketing.

From this point of view, the structure of the Patients First accreditation for a patient centered healthcare provider was built following the European approach by dimensions, but also enriched with two key aspects of the US approach: (a) culture understood as a system of values that is carried and lived by employees, and (b) patient education and empowerment. For this reason, the certification includes a specific dimension on employee involvement and another dimension of patient empowerment.

Out of this structure based on a combination of the US and the European models, Patients First establishes seven dimensions that determine a hospital with a patient centered model.

1. Communication: information of quality that is understandable by patients. Validation of main communication outcomes by patients and following of doctor-patient communication guides or standards

2. Tools and methodologies: capturing and understanding of patient experience with professional tools and methodologies

3. Systematic improvement: systematic improvement of patient experience out of insights. Improvement protocols and methodologies that include patient involvement and co-creation

4. Empowerment of healthcare professionals: involvement of healthcare professionals in patient experience and support for them.

5. Empowerment and self-management of patients: development of policies, methodologies, technologies and protocols that allow patients to manage - at least partially - their condition.

6. Ethics: patient representation in an ethics committee before hospital management

7. Research: use patient centered research standards.

\section{Materials and Methods}

Each one of these dimensions has a checklist of points to evaluate from a triple perspective:

1. Document analysis (evidence presented by the centre)

2. Practical application checked with participant observation

3. Validation through patient perception using participatory healthcare workshops and later surveys based on insights

Guadarrama hospital was particularly suited for the testing. The former sanatorium for tuberculosis is situated 981 meters high in the mountains and $65 \mathrm{~km}$ away from Madrid. Nowadays, it works as a midsized clinic of medium term stay for functional and cognitive impairment. The fact that it had 3 simple recovery processes and a manageable size made it ideal for testing purposes. Further, the hospital had a good record of awards in quality and patient orientation that ensured that management and staff would be willing to value healthcare quality and patient wellbeing.

The evaluation team was composed by an expert in healthcare quality, a psychologist and an anthropologist, with previous experience as quality manager in a private fertility clinic on IEXP side and by nurses and physiotherapists on Guadarrama's side.

The dimension seven (research) was excluded from the evaluation protocol since Guadarrama does not undertake research projects nor clinical trials.

For all the other dimensions, the evaluation team investigated for five months all evidence presented by Guadarrama hospital for each dimension. The team made 5 participant observation sessions and also interviews with staff and patients during the observation time. In order to triangulate data, the team made an inquiry workshop with patients and another inquiry workshop with the employees.

The inquiry workshop with patients was intended to learn about the patient's perspective and experience on each of the dimensions in order to find out if and how the hospital's policies and measures were perceived by patients. The workshop also gave valuable insights on other points that were important to patients, such as medication administration or a better use of physiotherapy time. 
The employee workshop was meant to identify if staff was actually aligned with patient experience policies and practices and also to detect possible organizational barriers to patient centered care.

As an outcome of the triple verification (documentation, participant observation and direct patient and employee inquiry as described in the material and methods section), the hospital received a report valuating all the points of the checklist for each dimension, as well as strengths and weaknesses to be improved. The reported included also specific suggestions and areas to work on.

\section{Results}

The goals of the pilot aimed to test if the accreditation was feasible both in terms of structure and in terms of process, as well as to test the kind of outcomes it produced for patients and the organization.

Structuring patient experience in seven dimensions proved to be useful in order to organize the patient experience process by blocks or work packages that can be assumed easily by the organization, as the Guadarrama Hospital team stated. Also, when auditing the organization, it was easy to follow the blocks as auditing pathway.

Guadarrama employees found the structure easy to understand and manage. Yet, some criticism about the wording of dimension 2 was expressed (originally named as "in depth understanding and analysis of patient needs"), so it was reformulated as "capturing and understanding of patient experience with professional tools and methodologies."

The pilot showed that there was one important dimension missing in the theoretical design of the accreditation, which is the emotional support for patients. Patients, employees and also one of the consultants pointed separately at different stages of the pilot that this aspect was missing. This idea emerged also in discussions during the employee experience workshop that is described below.

For the way the audit process was conducted, the hospital management valued positively both participant observation and the workshops conducted with patients and employees as a source of information and insights. The hospital team stated that the patient's inquiry workshop could have been used as a basis for a quantitative survey.

The employee workshop is a cornerstone of the accreditation, since patient experience depends to an important extent on employee experience. ${ }^{30,31}$ The purpose of the workshop is to ensure employee involvement and empowerment (dimension 4) and also align healthcare professionals with the dimensions of the accreditation and to allow management to understand and remove the barriers for this alignment. The workshop was seen at the beginning with caution, since management feared that possible employee discontent could have a negative impact on the project. At the time of this pilot, public healthcare in Spain was suffering from heavy budgetary cuts and the working climate in many hospitals was bad. These fears proved to be unfounded. Discontent arose as a matter of fact. But instead of leading to conflict, it was being expressed in an arena not meant for dispute or negotiation and it could thus be channeled to management in a more collaborative way. The workshop allowed most healthcare professionals to remember the vocational nature of their work and helped them to propose creative solutions for a better patient experience. In this sense, the employee experience workshop fulfilled its goals of staff alignment and management understanding.

Regarding the kind of outcomes produced by the process of evaluating patient experience with the Patients First tool, there were short, mid and long-term effects. Guadarrama Hospital implemented four immediate shortterm improvement measures. First, improvement of the signalization of admission at the ambulance entrance. Second, a review of the hospital's signage from patient's point of view with patient engagement techniques. Third, the introduction of training in patient journey mapping for the staff. Fourth, initiation of a patient involvement project in the valuation and registration of the documentation addressed to patients.

In the mid-term, Guadarrama opened 8 improvement areas as a direct result of the accreditation project.

- Analyzing all procedures and protocols of the quality management system to allow patient engagement.

- New ways to evaluate the information addressed to patients, like universal patient language and use of the inflesz scale for readability

- Patient centered design approach for web improvement

- Shared decision making

- Self-care for healthcare professionals

- Involvement of patient associations and families in the hospital

In the long term, Guadarrama Hospital established as an outcome of the accreditation a patient experience process based on three steps: capturing, transforming and measuring patient experience in a systematic way. A specific person was appointed as responsible of conducting and managing the process.

Regarding economic impact, some calculations were made in relation to some of the measures. For instance, the new 
signalization code saved 15 minutes per patient in admission, which makes 250 hours a year. There are also some possible correlations that could link the effects of improvements on cost. For instance, the training in patient journey mapping was applied to a program on better sleep for patients, with fewer interruptions and less noise that had a consequence on complaints and the time dedicated to their management. Also, the involvement of patients and family members in information co-design seemed to have some impact on stress and anxiety levels. Yet, there is not enough evidence about these correlations, and therefore the project showed no clear link between accreditation and costs.

The above-described points show that the application of the accreditation itself had a positive impact both for patients and for the hospital, since it produced improvements in patient experience in the short, mid and long term. The most important of them is surely the definition of a patient experience process with an appointed responsible person.

\section{Discussion}

The goal of the pilot project was to test if the accreditation structure in dimensions and the certification process were feasible and produced meaningful outcomes for patients and the Guadarrama Hospital. An insightful article by Jaafaripooyan, Agrizzi and Akbari-Haghighi ${ }^{32}$ on performance measures that allow the evaluation of healthcare accreditation systems states that perception of healthcare professionals regarding the outcomes of accreditations should focus: first, on the impact of accreditation on the quality and safety of healthcare delivery; second, on the efficiency of accreditation tools and systems for providing feedback with reliable information both to the accreditation organizations as well as all key stakeholders; and third, on the impact on the capacity development of systems.

Using this perspective might be useful to assess the outcomes of the Patients First accreditation. The structure of the accreditation following the European dimension approach is meant to ensure that healthcare providers set up a patient experience process that allows them to listen, transform, empower and measure patient experience. Thus, this structure helps hospitals to become patientcentric and focus on the questions that matter to patients, such as ensuring continuum of care, allow family involvement or ensuring physical comfort. ${ }^{33}$ But it does not aim to give a comprehensive list of all possible dimensions of patient experience, since care and patient experience can be very diverse depending on conditions (for instance, the care approaches and the experience in dealing with cancer ${ }^{34}$ is completely different than in dealing with mental health. ${ }^{35}$ ) It is also not oriented towards a series of "must have" points of patient experience, like for instance the NHS framework for Patient Experience, ${ }^{28}$ but it is rather conceived as a pathway and set of tools that enables the organization to define its own patient experience framework. The ultimate goal of this flexibility is to allow different kinds of healthcare organizations to use the tool in a strategic way according to their nature and goals.

The certification process is aimed to triangulate three information sources: (a) proceedings and documentation, (b) evaluators' observations and (c) patient's and employees' perceptions. This process is designed in order to obtain direct feedback on stakeholders' perception of those actions that are documented in proceedings. This allows the identification of possible gaps between theory and practice and it also reduces the frequent criticism on excessive bureaucracy of healthcare accreditations. ${ }^{36,37,38}$

Many studies and reviews assess healthcare accreditation processes as initiatives that have a positive impact fostering quality improvement, organizational change and learning. ${ }^{36,39-41}$ On the negative side, reviews often also mention a possible results an increase of bureaucracy and - somewhat less frequently - risks of time pressure, increases in working hours, and negative impacts on work life balance and on patient's care. ${ }^{38}$ In the case study reported in this communication, the collected evidence has been positive overall: the accreditation process led to the opening of eight new improvement areas for patients. It is significant to consider that the Guadarrama Hospital had already a record in humanization policies and patient satisfaction improvement projects, yet those efforts had not identified the potential areas of improvement that the accreditation uncovered, as the hospital's team ensured. This is not only relevant from a quantitative point of view, but also from a qualitative point of view, since it includes complex issues, like involvement of families, caregivers and patient associations, of shared decision-making. The project seems to suggest that an accreditation process in patient experience contributes to an improvement of results both for patients and the healthcare organization.

In the light of Jaafaripooyan's approach to performance measures for the evaluation of healthcare accreditation systems, the Patients First accreditation has produced an increase in quality with the short, mid and long-term measures undertaken by Guadarrama Hospital. It is efficient at providing stakeholder's feedback in two ways: during the evaluation process thanks to the patient and employee workshops and also in the longer term as a result of the introduction of patient experience capture tools.

\section{Tool enhancements}

Also, the pilot experience at Guadarrama showed the necessity of various enhancements of the accreditation itself. 
First, the pilot proved that emotional support for patients, caregivers but also for healthcare professionals is of great importance, since it correlates with quality of life and better prognosis, as the work of $\mathrm{CanO}^{42}$ and Adamson ${ }^{43}$ show. Also, Reinares-Lara Rodriguez-Fuertes and GarcíaHenche write about the double dimension of patient experience -cognitive and affective - and about the fact that "the affective or emotional dimension has been relegated to the background. For this reason, it seems appropriate now to incorporate this dimension." 44 Following the evidence as well as the results of the pilot, the accreditation has to include emotional support as one of its dimensions.

Second, the certification may be enhanced further by the inclusion of patients in its conceptual framework, according to WHO reccomendations ${ }^{45}$ and following also successful experiences of patient engagement in healthcare planning processes. ${ }^{46}$ It would be also positive to include the voice of a medical society specialized in quality.

Third, the metrics related to the measure of patient experience are included in dimension three (improvement of patient experience), yet the evidence of impact in this study is still weak. The pilot showed the necessity of including Patient Reported Outcome Measures (PROMs), Patient Experience Outcome Measures (PREMs) and Value Based Healthcare indicators in order to assess the real effect of the new patient experience measures and initiatives of the hospital on actual patient experience.

Fourth, the qualitative information gathering using a patient workshop has proven to be useful and valuable, but insights should be scaled and quantified using a survey.

\section{Limitations}

This study could not assess the impact of the measures undertaken by Guadarrama Hospital on actual patient experience on a clinical level, quality of life and economic. A second study is needed to evaluate the effect of those measures on patient experience. A second limitation consists in the fact that the need for emotional support as one of the dimensions was not considered in the initial design of the study. A review of the accreditation concept is needed in order to include emotional support and to assess the impact on actual patient experience. And a third limitation consists in the fact that the study was done in only one hospital. More testing studies are needed in order to extract broader conclusions, be able to compare data and define a standard for the accreditation.

\section{Conclusions}

The Patients First certification as a tool to evaluate if a hospital or clinic is patient centered seems to be robust from a conceptual and operative point of view. The methodology has proven to be useful to set up new working areas to improve patient experience, even in a hospital that has clear policies and good practices in this sense.

The proceeding requires improvement in engaging patients in the design, including PROMs, PREMs to assess the effect patient experience and a Value Based Healthcare Approach to better understand economic impacts. It is also needed to adopt a specific dimension in emotional support for patients and caregivers.

\section{References}

1. Doyle C, Lennox L, Bell D. A systematic review of evidence on the links between patient experience and clinical safety and effectiveness. BMJ Open. 2013;3(1):e001570-. doi:10.1136/bmjopen-2012001570

2. Rathert C, Wyrwich MD, Boren SA. Patient-Centered Care and Outcomes. Med Care Res Rev. 2013;70(4):351-379. doi:10.1177/1077558712465774

3. Mira JJ, Nuño-Solinís R, Guilabert-Mora M, et al. Development and Validation of an Instrument for Assessing Patient Experience of Chronic Illness Care. Int J Integr Care. 2016;16(3):13. doi:10.5334/ijic.2443

4. Anhang Price R, Elliott MN, Zaslavsky AM, et al. Examining the role of patient experience surveys in measuring health care quality. Med Care Res Rev. 2014;71(5):522-554. doi:10.1177/1077558714541480

5. Elliott MN, Lehrman WG, Goldstein E, Hambarsoomian K, Beckett MK, Giordano LA. Do hospitals rank differently on HCAHPS for different patient subgroups? Med Care Res Rev. 2010;67(1):5673. doi:10.1177/1077558709339066

6. Herrin J, Mockaitis KG, Hines S. HCAHPS Scores and Community Factors. Am J Med Qual. 2018;33(5):461-471. doi:10.1177/1062860618765977

7. Alkhenizan A, Shaw C. Impact of Accreditation on the Quality of Healthcare Services: A Systematic Review of the Literature. Ann Sandi Med. 2011;31(4):407. doi:10.4103/0256-4947.83204

8. Suñol R, Vallejo P, Thompson A, Lombarts MJMH, Shaw CD, Klazinga N. Impact of quality strategies on hospital outputs. Qual Saf Health Care. 2009;18 Suppl 1(Suppl 1):i62-8. doi:10.1136/qshc.2008.029439

9. Patel G. Total Quality Management in Healthcare. MIDAS J - Total Qual Manag Healthc. Published online 2015. Accessed August 3, 2018.

file://C:/Users/usuario/Downloads/IJ_690_1_Tot alQualityManagementinHC.pdf

10. Desveaux L, Mitchell JI, Shaw J, Ivers NM. Understanding the impact of accreditation on quality in healthcare: A grounded theory approach. Int J Qual Heal Care. 2017;29(7):941-947. doi:10.1093/intqhe/mzx136 
11. Salazar de la Guerra R, Ferrer Arnedo C, Labrador Domínguez MJ, Sangregorio Matesanz A. Certificación de los servicios enfermeros. Norma UNE-EN-ISO 9001-2008. Rev Calid Asist. 2014;29(6):350-354. doi:10.1016/j.cali.2014.10.003

12. Andres EB, Song W, Song W JJ. Can hospital accreditation enhance patient experience? Longitudinal evidence from a Hong Kong hospital patient experience survey. BMC Health Serv Res. Published online 2019. https://bmchealthservres.biomedcentral.com/articles /10.1186/s12913-019-4452-z

13. Auras S GM. Patient experience data in practice accreditation--an international comparison. Int J Qual Heal Care. Published online 2010.

14. Lam, M., Figueroa, J., Feyman, Y., Reimold, K., Orav, E., Jha A. Association between patient outcomes and accreditation in US hospitals: observational study. BMJ. 2018;363.

https://www.ncbi.nlm.nih.gov/pmc/articles/PMC61 93202/

15. van Dongen AJCM, Nelen WLDM, IntHout J, Kremer JAM, Verhaak CM. e-Therapy to reduce emotional distress in women undergoing assisted reproductive technology (ART): a feasibility randomized controlled trial. Hum Reprod. 2016;31(5):1046-1057. doi:10.1093/humrep/dew040

16. Stanhiser J, Steiner AZ. Psychosocial Aspects of Fertility and Assisted Reproductive Technology. Obstet Gynecol Clin North Am. 2018;45(3):563-574. doi:10.1016/j.ogc.2018.04.006

17. Jacobson NC, Roche MJ. Current evolutionary adaptiveness of anxiety: Extreme phenotypes of anxiety predict increased fertility across multiple generations. J Psychiatr Res. 2018;106:82-90. doi:10.1016/j.jpsychires.2018.10.002

18. Gdańska P, Drozdowicz-Jastrzębska E, Grzechocińska B, Radziwon-Zaleska M, Węgrzyn P, Wielgoś M. Anxiety and depression in women undergoing infertility treatment. Ginekol Pol. 2017;88(2):109-112. doi:10.5603/GP.a2017.0019

19. Dancet EAF, D'Hooghe TM, Spiessens C, et al. Quality indicators for all dimensions of infertility care quality: consensus between professionals and patients. Hum Reprod. 2013;28(6):1584-1597.

doi:10.1093/humrep/det056

20. van Empel IWH, Aarts JWM, Cohlen BJ, et al. Measuring patient-centredness, the neglected outcome in fertility care: a random multicentre validation study. Hum Reprod. 2010;25(10):2516-2526. doi:10.1093/humrep/deq219

21. Israilov $\mathrm{S}$, Cho HJ. HISTORY OF MEDICINE How Co-Creation Helped Address Hierarchy, Overwhelmed Patients, and Conflicts of Interest in Health Care Quality and Safety. Vol 19.; 2017. www.amajournalofethics.org

22. Minian N, Noormohamed A, Zawertailo L, et al. A method for co-creation of an evidencebased patient workbook to address alcohol use when quitting smoking in primary care: A case study. Res Involv Engagem. 2018;4(1). doi:10.1186/s40900-018-0086-2

23. Kuipers SJ, Cramm JM, Nieboer AP. The importance of patient-centered care and co-creation of care for satisfaction with care and physical and social wellbeing of patients with multi-morbidity in the primary care setting. BMC Health Serv Res. 2019;19(1). doi:10.1186/s12913-018-3818-y

24. Halvorsrud K, Kucharska RFJ. Lecturer Katherine Adlington, Core Psychiatry Trainee Katja Rüdell, Research/Teaching Fellow Eva Brown Hajdukova, Research Fellow James Nazroo, Professor of Sociology Maria Haarmans. Lect Sociol Kamaldeep Bhui. Published online 2017:1-12. doi:10.1093/pubmed/fdz126

25. Wolf JA, Palmer S. Guiding Principles for Patient Experience Excellence; 2016. Accessed August 22, 2018. https://www.theberylinstitute.org/store/download.as px?id=FEAABABC-FD97-4A75-8DEC47FEB31A933B

26. Beryl Institute. About The Beryl Institute - The Beryl Institute - Improving the Patient Experience. Published 2017. Accessed September 1, 2018. https://www.theberylinstitute.org/page/Mission?\&h hsearchterms $=\% 22 \% 22$ community + of + practice $\% 22$ $\% 22$

27. Cleveland Clinic. About Office of Patient Experience | Cleveland Clinic. Published 2018. Accessed September 1, 2018. https://my.clevelandclinic.org/departments/patientexperience/depts/office-patient-experience/about

28. NHS. NHS Patient Experience Framework. Published online 2011. Accessed September 1, 2018. https://assets.publishing.service.gov.uk/government/ uploads/system/uploads/attachment_data/file/2151 59/dh_132788.pdf

29. Picker Institute. Principles of person centred care: Picker. Published 2018. Accessed September 1, 2018. http://www.picker.org/about-us/principles-ofpatient-centred-care/

30. Berman S. Why human resources policies and practices are critical to improving the patient experience. Patient Exp J. 2016;3(2). Accessed December 18, 2020. https:/ / pxjournal.org/cgi/viewcontent.cgi?article $=11$ $87 \&$ context $=$ journal

31. Buhlman N, Lee T. When Patient Experience and Employee Engagement Both Improve, Hospitals' Ratings and Profits Climb. Harv Bus Rev. Published online 2019. Accessed December 18, 2020. https://hbr.org/2019/05/when-patient-experienceand-employee-engagement-both-improve-hospitalsratings-and-profits-climb

32. Jaafaripooyan E, Agrizzi D, Akbari-Haghighi F. Healthcare accreditation systems: further perspectives 
on performance measures. Int J Qual Heal Care. 2011;23(6):645-656. doi:10.1093/intqhc/mzr063

33. Foo C De, Tan YL, Shrestha P, et al. Exploring the dimensions of patient experience for communitybased care programmes in a multi-ethnic Asian context. PLoS One. 2020;15(11):e0242610. doi:10.1371/journal.pone.0242610

34. Bonacchi A, Di Miceli S, Lippi D, Muraca MG, Miccinesi G. Unmet needs of Italian cancer patients in different stages of the disease and care process. Tumori. 2018;104(4):285-291. doi:10.5301/tj.5000637

35. Mjsund NH, Eriksson M, Espnes GA, Vinje HF. Reorienting Norwegian mental healthcare services: Listen to patients' learning appetite. Health Promot Int. 2019;34(3):541-551. doi:10.1093/heapro/day012

36. Carrasco-Peralta JA, Herrera-Usagre M, ReyesAlcázar V, Torres-Olivera A. Healthcare accreditation as trigger of organisational change: The view of professionals. J Healthc Qual Res. 2019;34(2):59-65. doi:10.1016/j.jhqr.2018.09.007

37. Touati N, Pomey MP. Accreditation at a crossroads: Are we on the right track? Health Policy (New York). 2009;90(2-3):156-165. doi:10.1016/j.healthpol.2008.09.007

38. Alshamsi AI, Thomson L, Santos A. What Impact Does Accreditation Have on Workplaces? A Qualitative Study to Explore the Perceptions of Healthcare Professionals About the Process of Accreditation. Front Psychol. 2020;11. doi:10.3389/fpsyg.2020.01614

39. Greenfield D, Pawsey M, Hinchcliff R, Moldovan M, Braithwaite $\mathrm{J}$. The standard of healthcare accreditation standards: A review of empirical research underpinning their development and impact. BMC Health Serv Res. 2012;12(1). doi:10.1186/1472-696312-329

40. Alkhenizan A, Shaw C. Impact of accreditation on the quality of healthcare services: A systematic review of the literature. Ann Saudi Med. 2011;31(4):407-416. doi:10.4103/0256-4947.83204

41. Lanteigne $G$, Bouchard C. Is the introduction of an accreditation program likely to generate organizationwide quality, change and learning? Int J Health Plann Manage. 2016;31(3):e175-e191. doi:10.1002/hpm.2314

42. Cano Giménez E, Sánchez-Luna M. Providing parents with individualised support in a neonatal intensive care unit reduced stress, anxiety and depression. Acta Paediatr. 2015;104(11):e476-e476. doi:10.1111/apa.13135

43. Adamson K, Bains J, Pantea L, Tyrhwitt J, Tolomiczenko G, Mitchell T. Understanding the Patients' Perspective of Emotional Support to Significantly Improve Overall Patient Satisfaction. Healthc Q. 2012;15:63-69. doi:10.12927/hcq.2012.23193
44. Reinares-Lara P, Rodríguez-Fuertes A, GarciaHenche B. The Cognitive Dimension and the Affective Dimension in the Patient's Experience. Front Psychol. 2019;10(SEP):2177. doi:10.3389/fpsyg.2019.02177

45. World Health Organisation. Patient Engagement.; 2106.

46. Khodyakov D, Stockdale S, Smith N, Booth M, Altman L, Rubenstein L. Patient engagement in the process of planning and designing outpatient care improvements at the Veterans Administration Healthcare System: Findings from an online expert panel. Health Expect. 2016;20. doi:10.1111/hex.12444 